
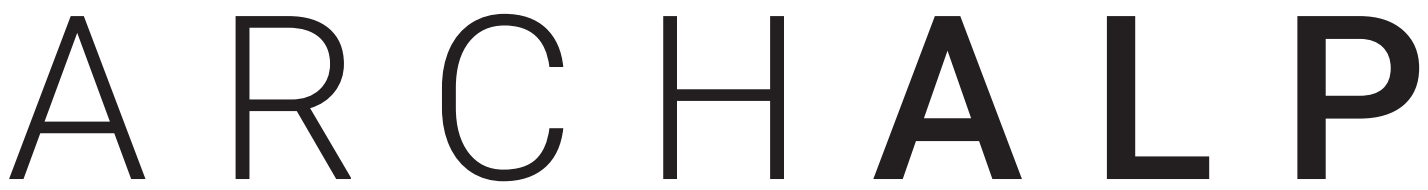

Rivista internazionale di architettura e paesaggio alpino / Revue internationale d'architecture et de paysage dans les Alpes / Internationale Zeitschrift für Alpine Architektur und Landschaft / Revija za alpsko arhitekturo in pokrajino / International journal of alpine architecture and landscape

Rer una nuota abitabilita delle Alpi.

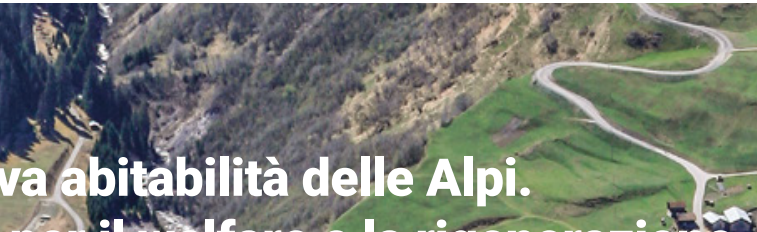
Architeture pet ilf lfare e la rigenerazione For a new inhabifability of the Alps. Architectures for welfare ând regeneration $/$ Pour une nouvelle habitabilité des Alpes. Architectures pour le welfare et la régéhérationy Für eine núue Bewohnbarkeit in den Alpen. Architekturen für Wohf ahrt und Regeneration / Za novo. bivalnost v Alpah, arhitekture za dobrobit in regeneracijo
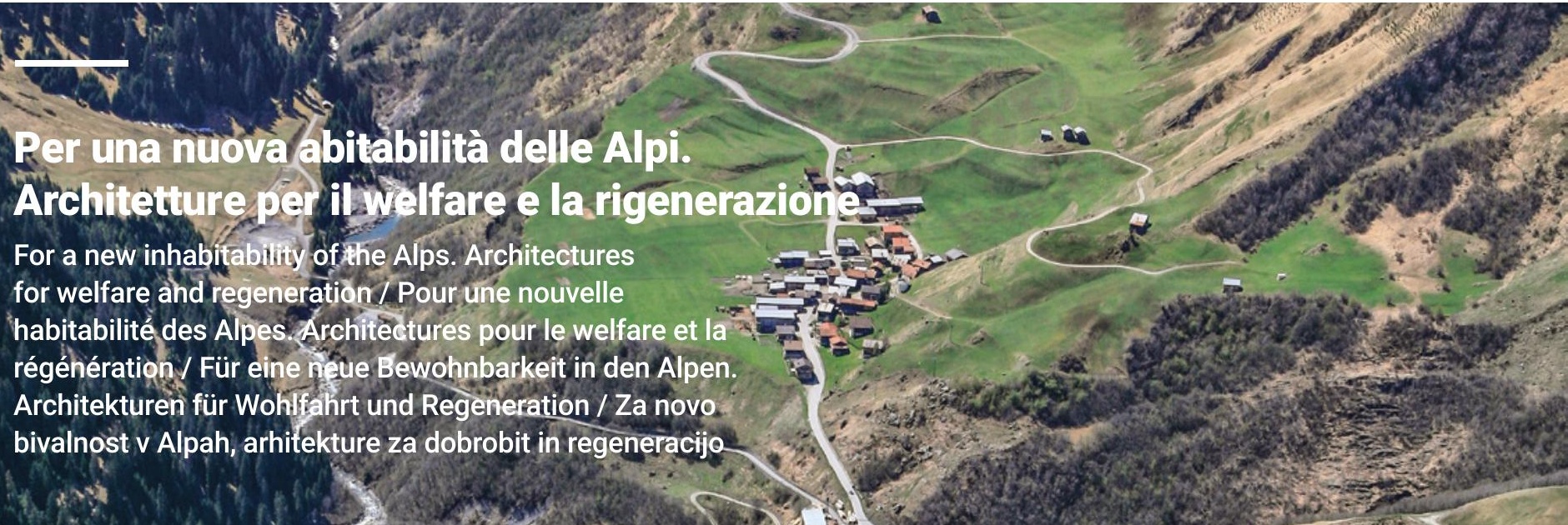
a $x \rightarrow 3$
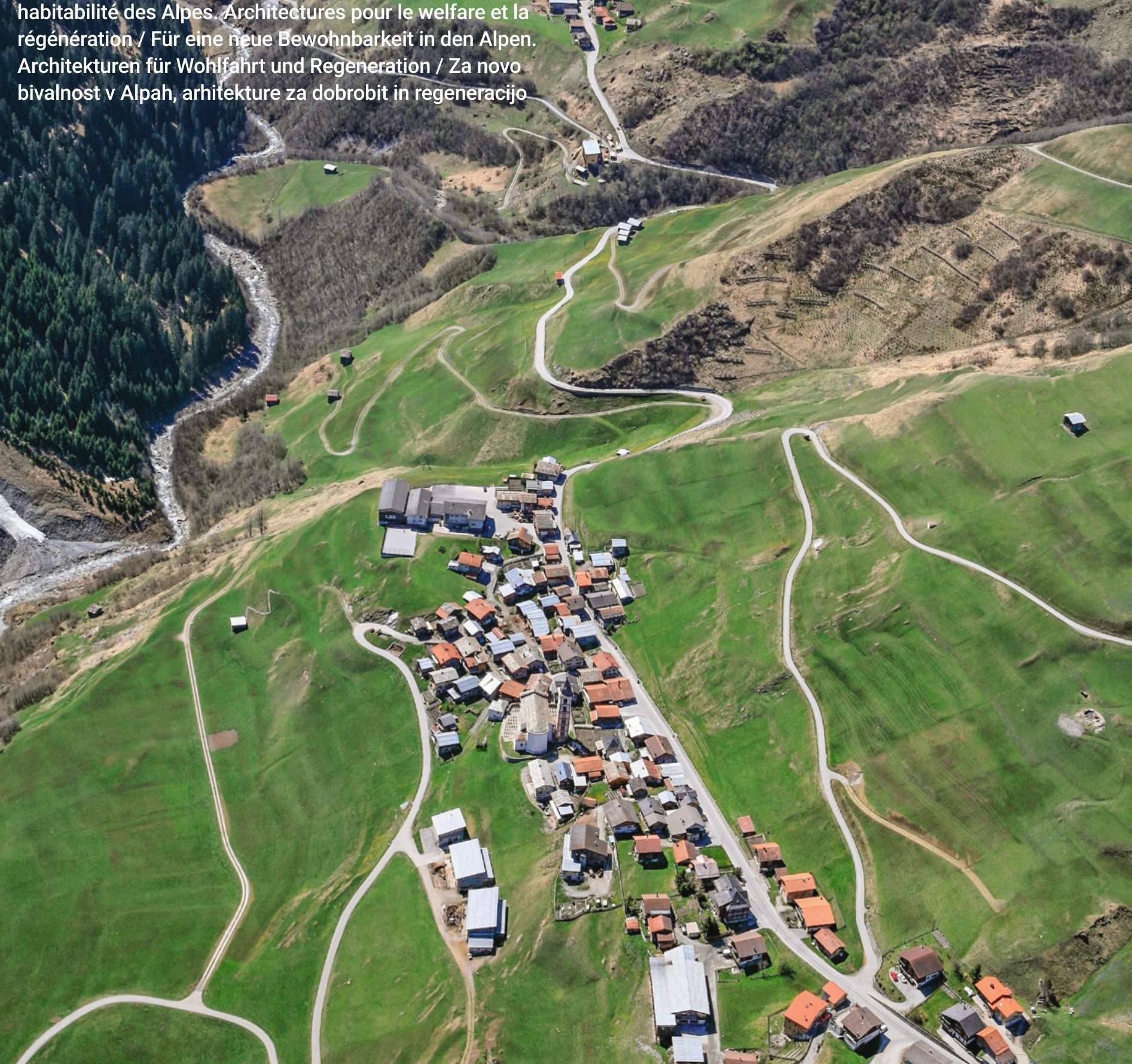


\section{Indice dei contenuti Contents}

\section{Temi}

Sulla centralità di spazio e territorio nel progetto di rigenerazione delle montagne e delle aree interne / On the centrality of space and territory in the project of regeneration of mountains and internal areas Antonio De Rossi, Laura Mascino

I servizi nelle Alpi italiane: quali e dove? Idee per uno scenario post-pandemico / Facilities in the Italian Alps: which ones and where? Ideas for a post-pandemic scenario

Giuseppe Dematteis

Manifesto di Camaldoli per una nuova centralità della montagna

Alla ricerca della distanza perduta. Rigenerare luoghi, persone e immaginari del riabitare alpino / In search of the lost distance. Regenerating places, people and images related to Alpine reinhabitation

Filippo Barbera, Andrea Membretti

Futuro e rigenerazione

Marco Bussone

\section{Esperienze}

Dorferneuerung zwischen Erhalten und Gestalten / 
Valades ousitanes, architettura e rigenerazione / Valades ousitanes,

Ostana e Topolò: hardware, software e welfare nelle comunità di "ritorno" / Ostana and Topolò: hardware, software and welfare in "return" communities

Margherita Valcanover

"Senza mostrare i muscoli": i progetti di rigenerazione di Tao+C e AZL nel solco della "prosperosa società" della Cina contemporanea / "Without flexing one's muscles": the regeneration projects of Tao+C and AZL in the wake of the "prosperous society" of contemporary China Edoardo Bruno, Dalila Tondo

L'archipel Butor. Une régénération, par la culture, d'un village soumis à la métropolisation genevoise / The Butor archipelago. A regeneration, through culture, of a village subject to the metropolisation of Geneva Arnaud Dutheil

Pratiche e progettualità di rigenerazione e welfare: il "Premio triennale Giulio Andreolli - Fare paesaggio" / Actions and projects of regeneration and welfare: the "Premio triennale Giulio Andreolli - Fare paesaggio" Giorgio Tecilla

Architetture e strategie per il welfare. Il caso di Brunico in Val Pusteria / Welfare architectures and strategies. The Bruneck case in Val Pusteria

Eleonora Gabbarini

Arhitektura oživlja / Architecture revives Kristina Dešman, Maja Ivanič

Si Crans-Montana meurt. Soigner le corps malade d'une station / If Crans-Montana dies. Taking care of the ailing body of a tourist resort Patrick Giromini 


\title{
Futuro e rigenerazione
}

\author{
Marco Bussone \\ Presidente nazionale Uncem (Unione dei Comuni, delle Comunità e degli Enti montani)
}

Siamo al centro di un processo che ancora ci vede timidi, un po' impacciati. L'abbiamo inaugurato dieci anni fa, forse più, dicendo che i nostri borghi alpini non potevano continuare a essere dimenticati. Presidente Uncem era Lido Riba, Assessore regionale alla Montagna Bruna Sibille. C'erano ancora 48 Comunità montane in Piemonte. Per gli appassionati di numeri, bisogna aggiungere tre zeri per identificare un potenziale numero di immobili sui quali intervenire. C'era allora il primo embrione dell'Istituto di Architettura Montana. Vivo e decisivo. Ci siamo scelti subito. Così nel 2008 la Regione Piemonte prima in Italia ha scelto di investire oltre 30 milioni di euro per rigenerare i borghi montani, i villaggi alpini e appenninici. Una grande novità copiata da tutte le Regioni italiane. Non riprendiamo qui tutta la storia. È stata scritta innumerevoli volte e registrata in diversi volumi e sul sito borghialpini.it.

A questo primo passo, ne sono seguiti altri. Il primo per concludere quei lavori in 35 cantieri di borghi, a causa anche di un bando difficilissimo e troppa burocrazia. Il secondo per guardare a tutte le iniziative attorno a borghi delle Alpi e degli Appennini, anche al di fuori del Piemonte, che si stavano muovendo, spinti da privati ed Enti locali. Il terzo per concludere una mappatura organica del "potenziale", che Uncem ha presentato a gennaio, descrivendo analiticamente oltre 4 mila borghi piemontesi. Un processo da estendere a tutto il Paese.

Non dobbiamo però perderci in dieci anni di buone pratiche e ottimi interventi. Tutti narrati on line e su tanti saggi. I punti fermi devono però essere chiari. Ne sintetizzo cinque. Sfide e urgenze per il patrimonio e la nostra montagna. Futuro.

La prima. Recuperare borghi e singoli immobili va molto bene, è utile. Ma prima dobbiamo capire a cosa e a chi servono. Molti borghi rivitalizzati negli ultimi dieci anni non sono vivi come avremmo voluto. Pezzi di Alpi occidentali, soprattutto in Piemonte continuano a spopolarsi. Contrastare l'abbandono con adeguate politiche è una necessità per il quale occorre unire ingredienti diversi. Quello della rigenerazione dei borghi è uno, ma deve essere oggetto di un pensiero e di una oppor- tuna pianificazione. Non si va a caso rigenerando qualsiasi cosa.

Qui viene alla mano un'altra urgenza. Investire fondi pubblici va bene ma bisogna evitare i bandi come quello fatto da Regione Piemonte nel 2018 con fondi del PSR. Troppo complesso (oltre 100 pagine), sei mesi di istruttoria, due canali di finanziamento che non servono certo a rivitalizzare luoghi per nuove comunità. Perché con 11 milioni di euro sono stati messi insieme progetti per la realizzazione e il miglioramento delle opere di urbanizzazione e degli spazi aperti a uso pubblico delle borgate montane e per strutture culturali-ricreative. Le poche risorse disponibili (che in avvio di PSR non erano state previste e che sono state inserite grazie a un'azione di lobby da parte di Uncem) vanno investite bene. Meglio. Vale per la nuova programmazione.

Terzo punto. Appunto, cosa facciamo nel settennato europeo 2021-2027. Se l'Europa vuole insistere sugli smart villages, dovrà capire che i nostri paesi e i nostri villaggi sono cosa diversa rispetto ad altri Paesi UE. Hanno natura architettonica, sociale, storica molto diversa rispetto ai villaggi delle campagne ungheresi e delle grandi distese francesi. I borghi alpini italiani sono diversi da quelli svizzeri e tedeschi. I Comuni hanno già fatto investimenti, ma per renderli smart c'è bisogno in primo luogo di colmare $\mathrm{i}$ divari digitali, portare nuovi servizi e opportunità. Soprattutto avere qualcuno che ci viva. Ecco la prima forma di smartness dei borghi. Viverli.

Per rigenerare secondo moderni stili, non chiudersi nel passatismo, non farlo tanto per dire che abbiamo ridato vita a quel borgo, servono strategie. Anche per fare investimenti privati che abbiano un senso di futuro e non finiscano contro un muro. Soldi ce ne sono pochi e trovare nuovi modelli di investimento privati richiede un pensiero attorno al modello economico. Per investire in un borgo farne un resort, ad esempio - non valgono i tempi di ritorno di un centro commerciale, di un outlet, di un albergone di periferia, come non ha senso immaginare che le banche possano farsi una risata (o quasi) quando si propone loro di individuare equity per un villaggio green $\mathcal{E}$ smart a 1300 metri 
di altitudine che deve rinascere grazie alla lungimiranza di uno o più imprenditori. È un quarto fronte difficilissimo che si affronta con un cambio di passo culturale degli istituti di credito, non certo capaci negli ultimi anni di andare incontro ai territori. Dai quali se ne sono andati con i loro sportelli. Che capiscano l'importanza - le banche - di una serie di investimenti su borghi e patrimonio dei territori, è da costruire.

Ultimo punto per il futuro (del nostro lavoro). Nei paesi delle valli, nei Comuni come nelle frazioni, dobbiamo ripensare i modelli e i modi di erogazione dei servizi. Scuola, trasporti, sanità. I tre pilastri della Strategia nazionale Aree interne. L'emergenza coronavirus ha riportato al centro il dibattito attorno all'organizzazione in particolare di servizi scolastici e sistema sanitario. Di certo nelle valli e nei territori rurali il ripensamento è complesso. Ripensare senza togliere. Potenziare senza rendere più rarefatto. Addensare senza costruire sistemi complessi dove è rimasto nessuno. Sulla scuola, ho sempre visto nelle "scuole di valle" una preziosa risposta. E nelle "case della salute" - unite a piattaforme per il volo notturno dell'elisoccorso e a potenziamento della presenza di medici di base - un antidoto all'eliminazione dei piccoli ospedali. Non ovunque però. Sagomare l'organizzazione dei servizi è il grande compito della politica. Come questi si riconducono sui territori è frutto di scelte che hanno ben poco a che fare con demagogia, social, facile esposizione mediatica. La complessità è evidente. E la politica la tiene lontana, di questi tempo.

Va di pari passo, questa urgenza, la quinta, con le altre precedenti quattro sfide. Programmare e pianificare sono compiti della politica che deve trovare risorse. Pubbliche prima di tutto, ma anche private, come abbiamo detto. Entrano in gioco modelli di presenza dello Stato diversi che abbiamo finora solo sperimentato in qualche best case nato dalla Snai o da altri percorsi di territorio. Di certo il pensiero non può essere municipale. Riorganizzare i servizi va oltre i confini amministrativi. Riusciremo a dare la giusta dimensione ai campanili e costruire attenzioni sulle politiche di territorio? Di nuovo entra in gioco la politica che usa le migliori teste e capacità per definire politiche per la montagna. Le abbiamo chieste - le politiche con una Piattaforma che è nata dagli Stati generali della Montagna convocati a fine gennaio dal Ministro Boccia a Roma. E che prende spunto dalle mozioni di tutti i partiti approvate all'unanimità a Montecitorio il 29 gennaio. Il percorso di rigenerazione dei borghi nasce in questo nuovo scenario che deve vedere la politica concretizzare proposte e istanze già varate dal Parlamento. Apriamo una fase nuova che non può essere ridotta a pensiero. Deve farsi azione, per i territori e le comunità che vogliono restare, tornare. 